\title{
The Surgical Specimen Is the Personalized Part of Personalized Cancer Medicine
}

\author{
Carolyn C. Compton, MD, PhD \\ Office of Biorepositories and Biospecimen Research, National Cancer Institute, Bethesda, MD
}

As a pathologist, I have been evaluating cancer resection specimens throughout my career and have come to have a deep appreciation of the challenges of surgical oncology. Happily for patients, surgeons cure cancer on a fairly regular basis by excising it with expert technique and detailed knowledge of human anatomy and tumor biology. The tissue resected by the surgeon and analyzed by the pathologist is the source of crucial information that, in turn, informs the decisions and actions of our medical oncology colleagues.

As cancer medicine looks forward to a new era of molecularly defined cancer subtypes and targeted therapies, however, the role of both surgeon and pathologist is evolving to require an ever greater degree of professional attention towards the surgical resection specimen. It is the surgically resected tissue that possesses the molecular information needed to define the specific molecular characteristics of the patient's tumor, the specific therapies to which the tumor would be expected to respond, and even the specific risks of adverse reactions to given therapies predicted by the patient's genetic make-up. This molecular information forms the basis of the "personalized" approaches envisioned for cancer patients in an age of molecular medicine. The professional responsibility to assure that the specimen's molecular composition and integrity are safeguarded is shared by both the surgeon and the pathologist. Current momentum towards molecular medicine is rapidly elevating this professional responsibility to one of the most important aspects of cancer patient care.

Currently, however, safeguarding the molecular integrity or documenting surgical variables that impact the molecular composition of the resection specimen is not

(C) Society of Surgical Oncology 2009

Published Online: 27 May 2009

C. C. Compton, $\mathrm{MD}, \mathrm{PhD}$

e-mail: comptcar@mail.nih.gov widely considered to be primary aspects of the surgeon's professional responsibility. Manipulations of the tissue within the surgical procedure itself may have dramatic effects on the molecular make-up of that tissue. However, these manipulations are neither recorded nor controlled when and where possible. Variables such as anesthesia type and duration, drugs administered preoperatively and intraoperatively, and devascularization/ischemia time for the resected tissue may dramatically alter molecular profiles and/or molecular integrity. Once successfully resected, the specimen may spend varying amounts of time at room temperature in the surgical suite and/or holding unit before being delivered to Pathology, which may further alter the molecular composition and quality of the tissue.

Without the surgeon's extension of professional responsibility to the resected tissue to control, when feasible, and track such variables, the advantages of personalized adjuvant approaches may be lost to the patient. In molecular medicine, the resected tissue becomes the major determinant of all downstream therapy. Therefore, the care of the specimen must be addressed co-equally with the care of the patient. This elevated bar for ensuring tissue integrity and molecular quality also must be addressed by pathologists. The fresh specimen will need to be overseen by the pathologist with the same immediacy and professional attention. More than ever, surgeons and pathologists will be required to work together closely to achieve the goal of meeting the new standards of "specimen care" required for molecular analysis.

Our knowledge about the affects of iatrogenic variables such as surgical manipulation, intraoperative drug delivery, and pathological handling on the molecular profiles that reflect the biology in resected tissues is growing rapidly. ${ }^{1-5}$ Postoperative tissue ischemia time, for example, has been shown to alter gene and protein expression profiles within minutes following surgical excision in colectomy specimens 
and prostatectomy specimens. ${ }^{1-3}$ Not surprisingly, even before resection has been completed, intraoperative manipulations have been shown to markedly alter gene transcription levels during radical prostatectomies. ${ }^{4}$ The effects of different peri- and intraoperative variables on molecular profiles in different types of tissues are just beginning to be understood, but it is clear that surgeons and pathologists alike contribute significantly to the final molecular composition and integrity of the resected tissue. ${ }^{5}$

Procedures that maximize specimen quality respect the fact that resected tissues are vital and biologically reactive. Until they are fixed or frozen, biospecimens are viable and capable of reacting to physiological stress. They are a living part of the patient from which they come and are responsive to changes in temperature, perfusion, oxygenation, and other physiological and biochemical variables, both pre- and intraoperatively as well as postoperatively. Typically, once a tumor is successfully resected, the surgeon's attention turns to patient and relatively little is directed towards the specimen. Unless an intraoperative consultation such as a frozen section is requested and the specimen is immediately addressed for this purpose, it may sit unattended for varying periods of time before being prepared for delivery to the pathology laboratory. The conditions of delivery itself may vary, as may the immediacy of the specimen handling once it has arrived in the pathology laboratory. Furthermore, some of the newer surgical techniques, such as robotically assisted prostate resections, may further compromise the quality of the resection specimen if it is allowed to remain in the operative site at body temperature for significant amounts of time after devascularization.

In this emerging age of molecular medicine, a new level of awareness of and attention to "the state of the specimen" will be required by surgeons, operating room staff, pathologists, and pathology staff. All play essential roles in the series of events leading up to stabilization of the tissue that impact its molecular make-up and molecular integrity. Surgeons are the initiators and controllers of many of these events and therefore represent the "gatekeepers." The powerful molecular analysis technologies now at our disposal and the increasingly sensitive and specific analysis platforms under development provide us with unprecedented abilities to define the molecular features of cells and tissues. However, they also pose new risks by providing us with the ability to derive the wrong answer with even greater speed and accuracy unless the analytes are of high quality and are derived from high-quality specimens. It is our joint professional responsibility to follow procedures that will ensure the quality of the biospecimen and to document the specimen handling history for the patient's record.

In this dawning era of molecular medicine, where a hard-won understanding of the molecular details of cancer is leading to more powerful and accurate diagnostics and therapeutics, I foresee surgeons and pathologists playing a new and more pivotal role in cancer medicine. They are the custodians of the specimens and therefore the molecules that represent the personalized part of personalized medicine. Surgeons will continue to cure cancer with greater success rates through earlier detection and excision; but as the custodians of the tissue, they will also be central to improving cancer management through molecularly targeted interventions.

\section{REFERENCES}

1. Spruessel A, Steimenn G, Jung M, Lee SA, Carr T, Fentz A-K, et al. Tissue ischemia time affects gene and protein expression patterns within minutes following surgical excision. BioTechniques. 2004;30:1030-7.

2. Dash A, Maine IP, Varambally S, Shen R, Chinnaiyan AM, Rubin MA. Changes in differential gene expression because of warm ischemia time of radical prostatectomy specimens. Am J Pathol. 2002;161:1743-8.

3. Lin DW, Coleman IM, Hawley S, Huang CY, Dumpit R, Gifford $\mathrm{D}$, et al. Influence of surgical manipulation on prostate gene expression: implications for molecular correlates of treatment effects and disease prognosis. J Clin Oncol. 2006;23:3763-70.

4. Schlomm T, Näkel E, Lübke A, Buness A, Chun FK-H, Steuber T, et al. Marked gene transcript level alterations occur early during radical protatectomy. Eur Urol. 2008;53:333-46.

5. Signoretti S, Bratslavsky G, Waldman FM, Reuter VE, Haaga J, Merino M, et al. Tissue-based research in kidney cancer: current challenges and future directions. Clin Cancer Res. 2008;14:3699705. 